January 6, 2022 11:12 WSPC/INSTRUCTION FILE "ws-jmm

Journal of Multiscale Modelling

() Imperial College Press

\title{
INTER-ELEMENT CRACK PROPAGATION WITH HIGH-ORDER STRESS EQUILIBRIUM ELEMENT
}

\author{
FRANCESCO PARRINELLO \\ Engineering Department, University of Palermo, Address \\ Palermo, 90128, Viale delle Scienze, Italy \\ IVANO BENEDETTI \\ Engineering Department, University of Palermo, Address \\ Palermo, 90128, Viale delle Scienze, Italy \\ Received (Day Month Year) \\ Revised (Day Month Year)
}

\begin{abstract}
The present contribution proposes a formulation based on the use of hybrid equilibrium elements (HEEs), for the analysis of inter-element delamination and fracture propagation problems. HEEs are defined in terms of quadratic stress fields, which strongly verify both the homogeneous and inter-element equilibrium equations and they are employed with interfaces, initially exhibiting rigid behaviour, embedded at the elements sides. The interface model is formulated in terms of the same degrees of freedom of the HEE, without any additional burden. The cohesive zone model (CZM) of the extrinsic interface is rigorously developed in the damage mechanics framework, with perfect adhesion at the pre-failure condition and with linear softening at the post-failure regime. After a brief review, the formulation is computationally tested by simulating the behaviour of a double-cantilever-beam with diagonal loads; the obtained numerical results confirm the accuracy and potential of the method.
\end{abstract}

Keywords: Fracture; extrinsic interface; hybrid equilibrium.

\section{Introduction}

The numerical modelling of nonlinear behaviours in brittle materials can be approached in the framework of continuum damage mechanics, by using suitable regularization techniques to avoid pathological mesh dependency, or by adopting discrete crack models, such as the zero-thickness interface element, strong or embedded discontinuity (EFEM) approaches 1,2,3,4 and the eXtended-generalized FEM (XFEM, GFEM) 5,6,7,8. Discrete crack models are often combined with Cohesive Zone Models (CZMs) 9,10,11,12,13,14,15,16,17,18,19,20, which describe the Traction Separation Law (TSL) at the discontinuity surface.

Most of the interface are modelled with an initial elastic behaviour which represents the approximation of the penalty method applied to keep together the two connected bodies along the joining surface. The initial elastic behaviour introduces an additional and artificial compliance which alters the elastic response and can produce pathological traction oscillations at the crack tip ${ }^{21}$. In order to overcome such numerical issues, an initial rigid 
behaviour has been modelled with the Discontinuous Galerkin (DG) method in Ref. ${ }^{22}$ and by the mixed formulation, based on the augmented Lagrangian method, in Ref. ${ }^{23}$, for the inter-element fracture propagation.

The formulation proposed in the present paper is developed in the same computational framework as that of the fracture propagation analysis at the inter-element interface, such as proposed in Refs. ${ }^{22,23,24,25}$, but it is defined through the equilibrium-based finite element formulation with embedded extrinsic interface at the element sides. The equilibriumbased formulation for elastic-dynamic analysis is developed in Ref. ${ }^{26}$. The proposed formulation provides a solution of the elastic problem with a quadratic stress fields that strongly verifies the homogeneous equilibrium equations and inter-element equilibrium equations $27,28,29,30,31,32,33,34$. The extrinsic interface is modelled by an initially rigid traction-separation law developed in the rigorous thermodynamic framework of damage mechanics.

In the Refs. ${ }^{22,23,24,25}$ the inter-element crack approach is effectively applied on prediction of crack path for a wide range of geometries and load conditions, both static and dynamic. The main drawback of the formulations based on the inter-element crack propagation, is the mesh dependency; however, this problem is strongly mitigated by mesh refinement and a future improvement of the proposed model could be the correction of the mesh geometry ahead of the crack tip, so that an element side is oriented along the maximum principal stress direction.

\section{The hybrid equilibrium element (HEE)}

In the present paper the hybrid equilibrium element is defined for a two-dimensional membrane problems with null body force by the quadratic stress fields satisfying the domain equilibrium equation. The triangular finite element of domain $\Omega_{e}$ is referred to a local Cartesian reference $\left(x_{1}, x_{2}\right)$ centred at vertex 1, as represented in Fig.1. The membrane stress fields are defined by the following quadratic polynomial functions

$$
\begin{gathered}
\sigma_{1}=a_{1}+a_{2} x_{1}+a_{3} x_{2}+2 a_{4} x_{1} x_{2}+a_{5} x_{1}^{2}+a_{6} x_{2}^{2}, \\
\sigma_{2}=a_{7}+a_{8} x_{1}+a_{9} x_{2}+2 a_{10} x_{1} x_{2}+a_{11} x_{1}^{2}+a_{5} x_{2}^{2}, \\
\tau_{12}=a_{12}-a_{9} x_{1}-a_{2} x_{2}-2 a_{5} x_{1} x_{2}-a_{10} x_{1}^{2}-a_{4} x_{2}^{2},
\end{gathered}
$$

where $a_{1}, \ldots, a_{12}$ are generalized stress variables. The stress fields of Eqs.(2-2) implicitly satisfy equilibrium equation for null body force $(\operatorname{div} \sigma=\mathbf{0})$ and can be represented in the following Voigt notation

$$
\boldsymbol{\sigma}=\mathbf{S} \cdot \mathbf{a}
$$

where a collects all generalized stress variables and

$$
\boldsymbol{\sigma}=\left[\begin{array}{c}
\sigma_{1} \\
\sigma_{2} \\
\sigma_{12}
\end{array}\right], \mathbf{S}=\left[\begin{array}{cccccccccccc}
1 & x_{1} & x_{2} & 2 x_{1} x_{2} & x_{1}^{2} & x_{2}^{2} & 0 & 0 & 0 & 0 & 0 & 0 \\
0 & 0 & 0 & 0 & x_{2}^{2} & 0 & 1 & x_{1} & x_{2} & 2 x_{1} x_{2} & x_{1}^{2} & 0 \\
0 & -x_{2} & 0 & -2 x_{2}^{2} & -2 x_{1} x_{2} & 0 & 0 & 0 & -x_{1} & -2 x_{1}^{2} & 0 & 1
\end{array}\right]
$$

The inter-element and boundary equilibrium conditions are imposed by a Lagrangian approach, and an independent Lagrangian variable (a displacement field) is considered for 
each element side. In order to strongly verify the inter-element equilibrium condition, with codiffusive stresses at any internal element side, the quadratic polynomial displacement fields, with three independent nodes for every element side, are considered. The HEE with quadratic stress field is represented in Fig.(1) and is governed by nine nodes, with two geometrically coincident nodes for each vertex. Displacement continuity is not assumed a priori. In fact, in the proposed stress-based approach, equilibrium conditions are strongly satisfied, whereas displacement continuity cannot be imposed. The geometry and displacement of the element sides $\Gamma_{s}^{e}$ with $s=1,2,3$ are modelled by a classic isoparametric mapping

$$
\begin{array}{r}
\boldsymbol{x}_{s}^{e}(\xi)=\mathbf{N}(\xi) \cdot \mathbf{x}_{s}^{e} \\
\boldsymbol{u}_{s}^{e}(\xi)=\mathbf{N}(\xi) \cdot \mathbf{u}_{s}^{e},
\end{array}
$$

where $\mathbf{N}(\xi)$ collects the shape functions and $\mathbf{x}_{s}^{e}$ and $\mathbf{u}_{s}^{e}$ respectively are the vector of nodal coordinates and the vector of kinematic degrees of freedom of side $\Gamma_{s}^{e}$.

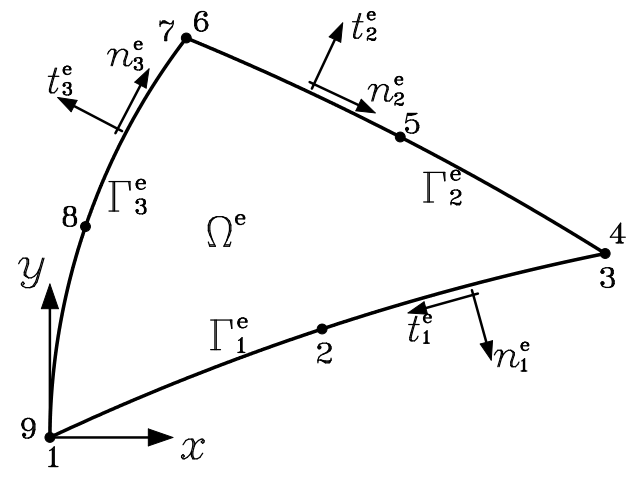

Fig. 1. Nine-node hybrid triangular finite element.

The element nodal force vector can be written as $\mathbf{q}_{e}=\mathbf{H}_{e}^{T} \mathbf{a}_{e}$ (see Ref. ${ }^{17}$ for details) and the equation of the single hybrid equilibrium element is

$$
\left[\begin{array}{cc}
\mathbf{C}_{e} & -\mathbf{H}_{e} \\
-\mathbf{H}_{e}^{T} & \mathbf{0}
\end{array}\right]\left[\begin{array}{l}
\mathbf{a}_{e} \\
\mathbf{u}_{e}
\end{array}\right]=\left[\begin{array}{c}
\mathbf{0} \\
-\mathbf{q}_{e}
\end{array}\right]
$$


where

$$
\begin{aligned}
& \mathbf{C}_{e}=\int_{\Omega_{e}} \mathbf{S}_{e}^{T} \mathbf{D} \mathbf{S}_{e} d \Omega, \\
& \mathbf{H}_{e}=\left[\mathbf{h}_{1}^{e}, \mathbf{h}_{2}^{e}, \mathbf{h}_{3}^{e}\right], \\
& \mathbf{h}_{s}^{e}=\int_{\Gamma_{s}^{e}} \mathbf{S}_{e}^{T} \mathbf{n}_{s}^{e} \mathbf{N} d \Gamma, \\
& \mathbf{T}_{e}=\left[\mathbf{t}_{1}^{e}, \mathbf{t}_{2}^{e}, \mathbf{t}_{3}^{e}\right], \\
& \mathbf{t}_{s}^{e}=\int_{\Gamma_{S}^{e} \cap \Gamma_{T}} \mathbf{N}^{T} \boldsymbol{t} d \Gamma, \\
& \mathbf{u}_{e}=\left[\mathbf{u}_{1}^{e}, \mathbf{u}_{2}^{e}, \mathbf{u}_{3}^{e}\right], \\
& \mathbf{n}_{s}^{e}=\left[\begin{array}{cc}
n_{s 1}^{e} & 0 \\
0 & n_{s 2}^{e} \\
n_{s 2}^{e} & n_{s 1}^{e}
\end{array}\right] \text {. }
\end{aligned}
$$

with $\mathbf{D}$ the constitutive elastic compliance matrix, $\mathbf{C}_{e}$ the element compliance matrix, $\mathbf{h}_{s}^{e} \mathbf{a}_{e}$ integrates traction on the element side $\Gamma_{s}^{e}, \mathbf{t}_{s}^{e}$ integrates the external forces $\mathbf{t}$ applied on element side $\Gamma_{s}^{e}$ of the free surface $\Gamma_{T}$ with outward normal $\mathbf{n}_{s}^{e}$. The compliance matrix $\mathbf{C}_{e}$ is symmetric, positive definite and is not singular, so that it can be inverted and the generalized stress can be condensed out as follows

$$
\begin{aligned}
& \mathbf{a}_{e}=\mathbf{C}_{e}^{-1} \mathbf{H}_{e} \mathbf{u}_{e} \\
& \mathbf{q}_{e}=\mathbf{H}_{e}^{T} \mathbf{C}_{e}^{-1} \mathbf{H}_{e} \mathbf{u}_{e}=\mathbf{K}_{e} \mathbf{u}_{e}
\end{aligned}
$$

where the matrix $\mathbf{K}_{e}=\mathbf{H}_{e}^{T} \mathbf{C}_{e}{ }^{-1} \mathbf{H}_{e}$ is the element stiffness matrix and the HEE can be implemented in a classic displacement-based finite element code. The possible presence of spurious kinematic modes can be approached by means of different available strategies $^{34,35,36,37}$.

\section{HEE with extrinsic interface embedded}

The hybrid equilibrium element with independent displacement fields for each side can model decohesion along an inter-elemet surface $\Gamma_{0}$, without any additional degree of freedom. The surface $\Gamma_{0}$ divides the two-dimensional elastic domain $\Omega$ into a positive part and a negative one, respectively $\Omega_{+}$and $\Omega_{-}$, as represented in Fig.2a. The behaviour of the connecting surface $\Gamma_{0}$ is modelled by an extrinsic interface and the relationship between traction $\mathbf{s}=\boldsymbol{\sigma}^{-} \mathbf{n}=-\boldsymbol{\sigma}^{+} \mathbf{n}$ and the separation displacement $[[\mathbf{u}]]=\mathbf{u}^{+}-\mathbf{u}^{-}$is defined as follows

$$
\mathbf{s}=\frac{1-\omega}{\omega} \mathbf{k}^{e l}[[\mathbf{u}]]
$$

where $\sigma^{ \pm}+$and $\mathbf{u}^{ \pm}$are respectively the stress and displacement of positive and negative sides of the interface $\left(\mathbf{x} \in \Omega_{ \pm} \cap \Gamma_{0}\right), \omega$ is the damage parameter and $\mathbf{k}^{e l}=\mathbf{I} k_{0}$ is the interface diagonal stiffness matrix, with $\mathbf{I}$ identity matrix and $k_{0}$ a stiffness parameter. The extrinsic interface produces an initial rigid behaviour for the pristine interface $(\omega=0)$ and null 


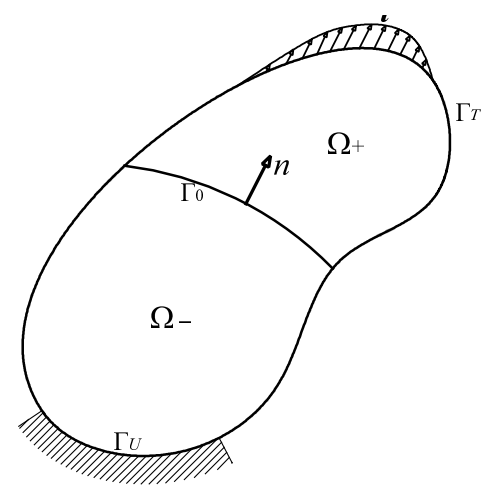

a)

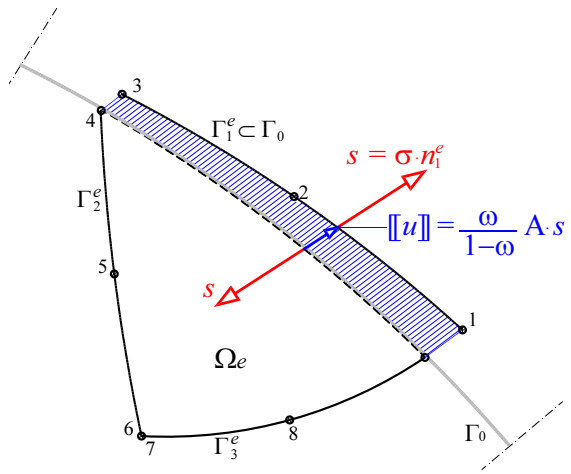

b)

Fig. 2. a) The elastic domains $\Omega$ divided by the extrinsic interface $\Gamma_{0}$ in the positive and the negative parts; b) quadratic hybrid equilibrium element $\Omega_{e}$ with an embedded extrinsic interface at side $\Gamma_{1}^{e}$.

traction at the fully debonding condition $(\omega=1)$. The interface can be embedded at the element side $\Gamma_{1}^{e}$ and the interface traction can be modelled as a function of the vector $\mathbf{a}_{e}$ of generalized stress variables of the HEE formulation, that is:

$$
\boldsymbol{s}(\mathbf{x})=\boldsymbol{\sigma}^{T} \mathbf{n}_{1}^{e}=\mathbf{a}_{e}^{T} \mathbf{S}^{T}(\mathbf{x}) \mathbf{n}_{1}^{e} \text { with } x \in \Gamma_{1}^{e} \subset \Gamma_{0} .
$$

The HEE formulation with embedded interface at element sides $\Gamma_{1}^{e}$ is governed by the following equation

$$
\left[\begin{array}{cc}
\mathbf{C}_{e}+\mathbf{C}_{e}^{\Gamma} & -\mathbf{H}_{e} \\
-\mathbf{H}_{e}^{T} & \mathbf{0}
\end{array}\right]\left[\begin{array}{l}
\mathbf{a}_{e} \\
\mathbf{u}_{e}
\end{array}\right]=\left[\begin{array}{c}
\mathbf{0} \\
-\mathbf{q}_{e}
\end{array}\right]
$$

where

$$
\mathbf{C}_{e}^{\Gamma}=\int_{\Gamma_{1}^{e}} \frac{\omega}{1-\omega} \mathbf{S}_{e}^{T} \mathbf{n}_{1}^{e} \mathbf{A}^{e l} \mathbf{n}_{1}^{e T} \mathbf{S}_{e} d \Gamma
$$

with $\mathbf{A}^{e l}=\mathbf{k}_{e l}^{-1}$. The matrix $\mathbf{C}_{e}^{\Gamma}$ is the additional compliance matrix due to the embedded interface and the matrix $\mathbf{K}_{e}=\mathbf{H}_{e}^{T}\left(\mathbf{C}_{e}+\mathbf{C}_{e}^{\Gamma}\right)^{-1} \mathbf{H}_{e}$ is the stiffness matrix of the HEE with embedded elastic interface. Eq.(6) can be derived by the stationarity condition of the complementary energy functional of the partially cracked solid, as proposed in Ref. ${ }^{17}$. In the proposed formulation the interface can be embedded at one or more sides of an hybrid equilibrium element without any additional degrees of freedom, simply considering the additional interface compliance matrix $\mathbf{C}_{e}^{\Gamma}$ defined in Eq.(7).

The behaviour of the interface is modelled in the rigorous thermodynamic framework of damage mechanics and the model is based on the following Helmholtz free energy function, defined for a unit of interface surface,

$$
\Psi=\frac{1}{2} \frac{1-\omega}{\omega}\left[[\boldsymbol{u}]^{T} \mathbf{k}^{e l}[[\boldsymbol{u}]\right.
$$


and the conjugated variable can be derived in the classic Coleman-Noll procedure as follows

$$
\begin{aligned}
Y & :=-\frac{\partial \Psi}{\partial \omega}=\frac{1}{2 \omega^{2}}[[\boldsymbol{u}]]^{T} \mathbf{k}^{e l}[[\boldsymbol{u}]] \\
\mathbf{s} & :=\frac{\partial \Psi}{\partial[[\boldsymbol{u}]}=\frac{1-\omega}{\omega} \mathbf{k}^{e l}[[\boldsymbol{u}]]
\end{aligned}
$$

where $Y$ is the energy release rate and $\mathbf{s}$ is the interface traction. The singularity condition of the latter Equation does not involve any numerical issue, in fact at the pristine condition $(\omega=0)$ separation displacement is null and inter-element interface is only modelled when the damage activation condition is attained. After the first damage increment $(0<\omega<1)$ the traction separation law in Eq.(3) is not singular.

\subsection{Damage activation condition}

In the thermodynamic framework, activation and evolution of damage are governed by the conjugated variable $Y$ and the damage activation function is defined as

$$
\phi_{d}(Y, \xi)=Y-Y_{0} \leq 0
$$

where $Y_{0}$ is the threshold for the first damage activation $(\omega=0)$, for which the energy release rate $Y$, defined in Eq.(3) in terms of displacement jump, is in an indeterminate form. However, for a perfectly bonded interface the energy release rate $Y$ can be written as a function of the traction by the substitution of Eq.(3) in Eq.(3), that is

$$
Y=\frac{1}{2} \frac{1}{(1-\omega)^{2}} \boldsymbol{s}^{T} \mathbf{A}^{e l} \boldsymbol{s} .
$$

Similarly, the energy release rate threshold can be defined as

$$
Y_{0}=\frac{s_{0}^{2}}{2 k_{0}}
$$

where $s_{0}$ is the interface strength. The evolution of damage variable is governed by the following associative flow rules and loading-unloading conditions

$$
\begin{aligned}
& \dot{\omega}:=\frac{\partial \phi}{\partial Y} \dot{\lambda_{d}}=\dot{\lambda}_{d} \\
& \dot{\lambda_{d}} \geq 0, \quad \phi_{d} \dot{\lambda_{d}}=0, \quad \dot{\phi}_{d} \dot{\lambda_{d}}=0 .
\end{aligned}
$$

with $\dot{\lambda}_{d}$ the damage multiplier. The proposed extrinsic CZM produces a bilinear traction separation law with a straight unloading-reloading branch and unlimited strength in compressive normal traction.

\section{Numerical simulation}

The formulation developed is implemented in FEAP code ${ }^{38}$ for a nine-node triangular HEE with quadratic stress fields and with extrinsic interface implicitly embedded at all element side. The numerical simulations of crack propagation test is performed for the 
double cantilever beam test with diagonal loads represented in the Fig.3a, with the FE discretization depicted in the Fig. $3 b$.

The experimental test was initially reported in Ref. ${ }^{39}$ and was analysed in Ref. ${ }^{40,41,42}$ by smeared crack and strong discontinuity approaches. Geometry and load setting of the experimental test are represented in Fig. 3a, the plate thickness is $50.8 \mathrm{~mm}$ and the constitutive parameters are the following: $E=30500 \mathrm{~N} / \mathrm{mm}^{2}, v=0.2$. The diagonal forces $\mathrm{F} 1$ increase until reaching $3.78 \mathrm{kN}$ and then are kept constant and the wedge forces F2 start to increase monotonically. In the numerical simulation presented, the wedge forces F2 are modelled as reaction forces of imposed separation displacement. In HEE, the concentrated force F1 at the upper right corner generates high stress level and, in order to avoid anomalous crack opening, the elements close to the corner are assumed as elastic.

The extrinsic interface is modelled with the following parameters $s_{0}=2 \mathrm{~N} / \mathrm{mm}^{2}$, $G_{I}=0.1 \mathrm{~N} / \mathrm{mm}$. The results of the numerical simulations are plotted in the Fig. 4 in terms of applied load $F 2$ and crack mouth opening displacement (CMOD) and are compared with the experimental data reported in Ref. ${ }^{39}$ and with the numerical results obtained by the XFEM formulation in Ref. ${ }^{42}$. The solution obtained by the proposed HEE formulation differs from the experimental data more than the XFEM solution. The reason of such larger error is probably related to the fact that inter-element crack formulations allow crack propagation only along inter-element interfaces, differently from XFEM, which does not suffer from such a constraint.

The maps of the maximum principal stress at the loading steps of CMOD values $\Delta u=$ $0.35 \mathrm{~mm}$ is plotted in the deformed configuration in the Figures $5 \mathrm{a}, \mathrm{b}$ with a detail of the inter-element crack.

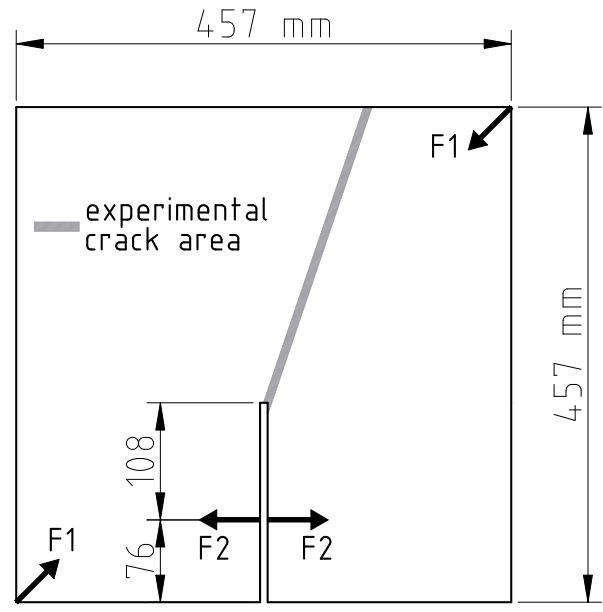

a)

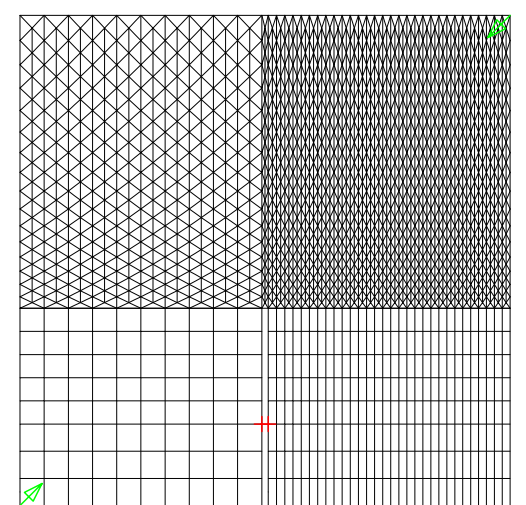

b)

Fig. 3. Double-cantilever-beam test with diagonal loads: a) sizes, geometry and loading condition; b) the FEM discretization. 
January $\quad 6$,

Parrinello-Benedetti"

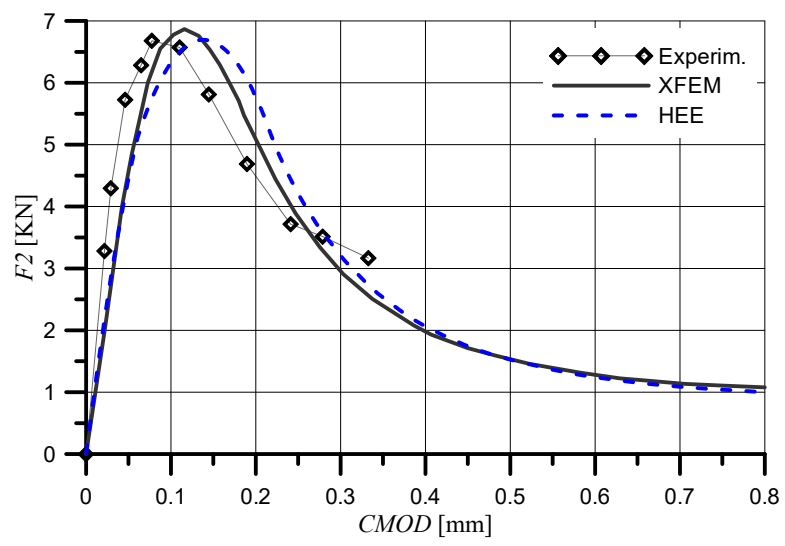

Fig. 4. Responses of the numerical simulations of the double-cantilever-beam test with diagonal loads in terms of load $F 2$ and crack mouth opening displacement (CMOD).

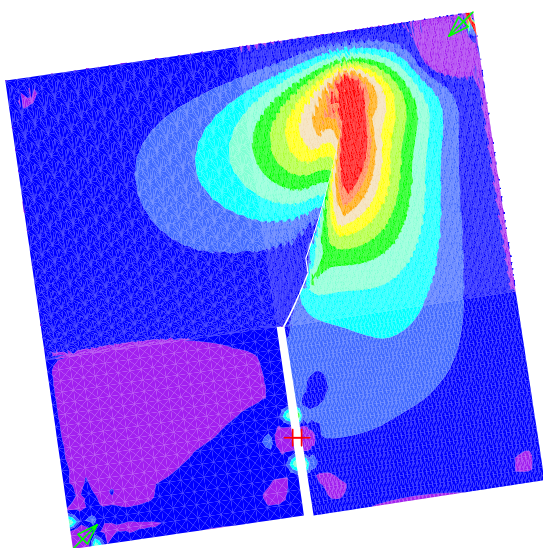

a)

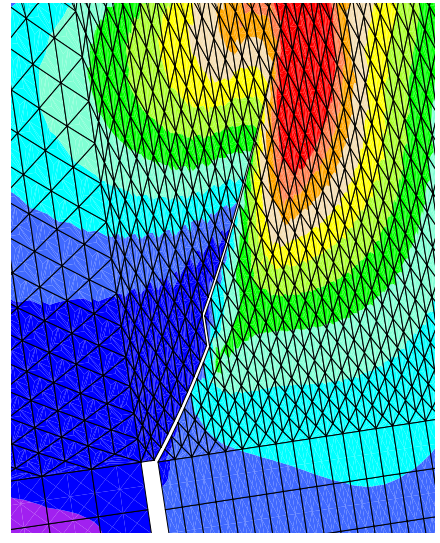

b)

Fig. 5. Maps of maximum principal stress $\sigma_{1}[\mathrm{MPa}]$, in the deformed configuration with the evolution of the crack, at the peak loading condition for: a) full mesh; b) detail of the inter-element crack.

\section{Closing remarks}

The paper presented an inter-element crack propagation model based on the use of Hybrid Equilibrium Elements with extrinsic interfaces embedded at the elements sides. The interface cohesive behaviour was developed in the rigorous thermodynamic framework of damage mechanics and produces a bilinear response with initially rigid behaviour. Future developments of the proposed model will be addressed at the formulation of HEEs for the dynamic analysis of fragmentation problems and for the correct evaluation of waves 


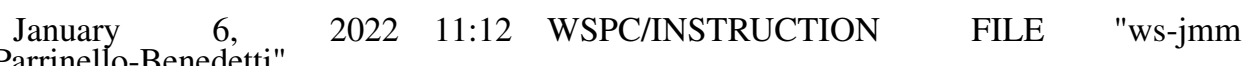

Parrinello-Benedetti"

Inter-element crack propagation with high-order stress equilibrium element 9

speeds.

\section{Acknowledgments}

The financial support of the Italian Ministry for University and Research (MIUR), under the Grant PRIN-2015, Project No. 2015LYYXA8, "Multiscale mechanical models for the design and optimization of microstructured smart materials and metamaterials" is gratefully acknowledged.

\section{References}

1. J. Simo and J. Oliver and F. Armero, An Analysis of Strong Discontinuities induced by strain softening in rate-independent inelastic solids, Comp.Mech., 12 p. 277-296 (1993).

2. M. Jirasek, Comparative study on finite elements with embedded discontinuities, CMAME, 188 p. 307-330 (2000).

3. J. Oliver and A.E. Huespe, Theoretical and computational issues in modelling material failure in strong discontinuity scenarios, CMAME, 193 27, p. 2987, (2004).

4. A. E. Huespe and J. Oliver, Crack Models with Embedded Discontinuities, Numerical Modeling of Concrete Cracking, (Hofstetter, Günter and Meschke, Günther, Springer, Vienna 2011).

5. N. Moës and J. Dolbow and T. Belytschko, A finite element method for crack growth without remeshing, Int.Jou.Num.Met.Eng. 46 1, p 131 (1999).

6. G. Zi and T. Belytschko, Crack Models with Embedded Discontinuities, Int.Jou.Num.Met.Eng., 57 15, p. 2221 (2003).

7. E. Benvenuti, A regularized XFEM framework for embedded cohesive interfaces, CMAME 197 49-50, p. 4367 (2008)

8. E. Benvenuti, An effective XFEM with equivalent eigenstrain for stress intensity factors of homogeneous plates, CMAME 321, p. 427, (2017).

9. A. Spada, and G. Giambanco and P. Rizzo, Damage and plasticity at the interfaces in composite materials and structures, CMAME 198 49-52, p. 3884-3901 (2009).

10. F. Parrinello and B. Failla and G. Borino, Cohesive-frictional interface constitutive model, Int. Jour. Solids Str., 46 13, p.2680-2692 (2009).

11. I. Benedetti and M.H. Aliabadi, A three-dimensional cohesive-frictional grain-boundary micromechanical model for intergranular degradation and failure in polycrystalline materials, CMAME, 265 p.36 - 62 (2013).

12. I. Benedetti and M.H. Aliabadi, Multiscale modeling of polycrystalline materials: A boundary element approach to material degradation and fracture, CMAME, 289, p.429 - 453, (2015).

13. F. Parrinello and G. Marannano and G. Borino, A thermodynamically consistent cohesivefrictional interface model for mixed mode delamination, Eng.Frac.Mech., 153 p. 61-79, (2016).

14. V. Gulizzi and I. Benedetti, Micro-cracking of brittle polycrystalline materials with initial damage, Eur.Jou.Comp.Mech., 25 1-2, p.38-53 (2016).

15. F. Parrinello, Analytical solution of the $4 \mathrm{ENF}$ test with interlaminar frictional effects and evaluation of Mode II delamination toughness, Journal of Engineering Mechanics, 144 4, (2018).

16. F. Parrinello and G. Borino, Non associative damage interface model for mixed mode delamination and frictional contact, European Journal of Mechanics / A Solids, 76 p.108-122 (2019).

17. F. Parrinello, Hybrid equilibrium element with interelement interface for the analysis of delamination and crack propagation problems, Int.Jou.Num.Met.Eng., 1221 p.190,218 (2021).

18. F. Parrinello and V. Gulizzi and I. Benedetti, A computational framework for low-cycle fatigue in polycrystalline materials, CMAME, 383 (2021). 
January 6, 2022 11:12 WSPC/INSTRUCTION FILE "ws-jmm

10 Parrinello, Benedetti

19. I.Benedetti and V.Gulizzi, A grain-scale model for high-cycle fatigue degradation in polycrystalline materials, International Journal of Fatigue, 116 p.90-105 (2018).

20. I.Benedetti and V.Gulizzi and A.Milazzo, A microstructural model for homogenisation and cracking of piezoelectric polycrystals, CMAME, 357 p.112595 (2019).

21. A. Simone, Partition of unity-based discontinuous elements for interface phenomena: Computational issues, Com.Num.Met.Eng., 20 6, p. $465-478$ (2004).

22. J. Mergheim and E. Kuhl and P. Steinmann, A hybrid discontinuous Galerkin/interface method for the computational modelling of failure, Com.Num.Met.Eng., 20 7, p. 511-519 (2004).

23. E. Lorentz, A mixed interface finite element for cohesive zone models, CMAME, 1982 p. 302 317 (2008).

24. R. Radovitzky and A. Seagraves and M. Tupek and L. Noels, A scalable 3D fracture and fragmentation algorithm based on a hybrid, discontinuous Galerkin, cohesive element method, CMAME 200 1-4 p. 326-344, (2011).

25. M.R. Hirmand and K.D. Papoulia, A continuation method for rigid-cohesive fracture in a discontinuous Galerkin finite element setting, Int.Jou.Num.Met.Eng., 115 5, p. 627-650 (2018).

26. F. Parrinello and G. Borino, Hybrid equilibrium element with high-order stress fields for accurate elastic dynamic analysis, Int.Jou.Num.Met.Eng., doi.org/10.1002/nme.6793, (preprint 2021).

27. B. Fraeijs de Veubeke, Stress Analysis, Zienkiewicz OC, Holister GS (eds), Chapter 9. Wiley: London (1965).

28. J.P.M. de Almeida and J.A.T de Freitas, An alternative approach to the formulation of hybrid equilibrium finite elements, Computers and Structures , 40 p. 1043-1047 (1991).

29. O.J.B. Almeida Pereira, Hybrid equilibrium hexahedral elements and super-elements, Com.Num.Meth.Eng., 24 p. 157-165, (2008).

30. J.P. De Moitinho Almeida and O.J.B.Almeida Pereira, A set of hybrid equilibrium finite element models for the analysis of three-dimensional solids, Int.Jou.Num.Met.Eng., 39, 16 p. 2789-2802 (1996).

31. M. Kempeneers and J.F. Debongnie and P. Beckers, Pure equilibrium tetrahedral finite elements for global error estimation by dual analysis, Int.Jou.Num.Met.Eng., 81 p. 513-536 (2010).

32. J.P.M.de Almeida and O.J.B.A.Pereira, A set of hybrid equilibrium finite element models for the analysis of threedimensional solids, Int.Jou.Num.Met.Eng., 39 p. 2789-2802 (1996).

33. O.J.B.A. Pereira and J.P.M. de Almeida and E.A.W. Maunder, Adaptive methods for hybrid equilibrium finite element models, CMAME , 176 p. 19-39 (1999).

34. F. Parrinello, Restraining approach for the spurious kinematic modes in hybrid equilibrium element, Comp.Mech., 524 p. 885-901 (2013).

35. E.A.W. Maunder and J.P. Moitinho de Almeida and A.C.A. Ramsay, A general formulation of equilibrium macro-elements with control of spurious kinematic modes: the exorcism of an old curse, Int.Jou.Num.Met.Eng., 39 p.3175-3194, (1996).

36. E.A.W. Maunder and J.P. Moitinho de Almeida, Hybrid-equilibrium elements with control of spurious kinematic modes, Computer Assisted Mech.Eng.Sci., 4 p. 587-605 (1997).

37. L. Wang and $\mathrm{H}$. Zhong, A traction-based equilibrium finite element free from spurious kinematic modes for linear elasticity problems, Int.Jou.Num.Met.Eng., 99 10, p.763-788 (2014).

38. O.C. Zienkiewicz and R.L. Taylor, The Finite Element Method. 5th Edition, ButterworthHeinemann Press, (2000).

39. A.S. Kobayashi and N.M. Hawkins and D.B. Barker and B.M. Liaw, Fracture process zone of concrete, NATO ASI Series, Series E: Applied Sciences, 94 p. 25-50 (1985).

40. J.G. Rots, Computational Modeling of Concrete Fractures, Delft University of Technology (1988).

41. J. Oliver and A.E. Huespe and M.D.G. Pulido and E. Chaves, From continuum mechanics to fracture mechanics: The strong discontinuity approach, Eng.Frac.Mech., 69 2, p.113-136 (2001).

42. J. Oliver and A.E. Huespe and P.J. Sánchez, A comparative study on finite elements for capturing 
January 6, 2022 11:12 WSPC/INSTRUCTION FILE "ws-jmm Parrinello-Benedetti"

Inter-element crack propagation with high-order stress equilibrium element 11 strong discontinuities: E-FEM vs X-FEM, CMAME, 195 37-40, p. $4732-4752$ (2006). 